\title{
On the dynamics of the three-piece-freight truck
}

\author{
Xia, Fujie; True, Hans
}

Published in:

Proceedings of the 2003 IEEE/ASME Joint Rail Conference, 2003.

Publication date:

2003

Document Version

Publisher's PDF, also known as Version of record

Link back to DTU Orbit

Citation (APA):

Xia, F., \& True, H. (2003). On the dynamics of the three-piece-freight truck. In Proceedings of the 2003 IEEE/ASME Joint Rail Conference, 2003. IEEE.

\section{General rights}

Copyright and moral rights for the publications made accessible in the public portal are retained by the authors and/or other copyright owners and it is a condition of accessing publications that users recognise and abide by the legal requirements associated with these rights.

- Users may download and print one copy of any publication from the public portal for the purpose of private study or research.

- You may not further distribute the material or use it for any profit-making activity or commercial gain

- You may freely distribute the URL identifying the publication in the public portal

If you believe that this document breaches copyright please contact us providing details, and we will remove access to the work immediately and investigate your claim. 


\title{
ON THE DYNAMICS OF THE THREE-PIECE-FREIGHT TRUCK
}

\author{
Fujie Xia and Hans True \\ Informatics and Mathematical Modelling \\ Richard Petersens Plads 321 \\ Technical University of Denmark \\ DK-2800 Lyngby, Denmark \\ phone: +4545253016 , fax; +454593 2373, e-mail: f.xia@cqu.edu.au and ht@imm.dtu.dk
}

\section{INTRODUCTION}

This paper is based on part of Fujie Xia's PhD-thesis [8] on the modelling and dynarnics of the Three-Piece-Freight Truck.

Although the three-piece-freight truck is a simple design its mathmatical model is very complicated. The model is definitely a nonlinear dynamical systern, where the nonlinearities arise from the nonlinear kinematic and dynamical contact relations between wheels and rails, the suspensions and the nonlinear dry friction damping with hysteresis and stick-slip action. The bolster moves both vertically and laterally relative to the truck frames, so the friction forces on the contact surfaces of the wedges must be treated as two-dimensional vectors, and the same holds for the dry friction on the surfaces of the adapters. Due to the clearances between the car body and the side supports on the bolster, the side supports must be modelled as nonlinear dead-band springs. The stick-slip action and the play between elements of the truck makes the dynamical model a structure varying system.

To our knowledge this model is the most detailed model of the three-piece-freight truck in existence today. The model consists of 19 rigid bodies including the masses of the wedges, and the total number of degrees of freedom of the system is 81. The most important novelty is the coupling between the vertical and lateral dynamics in the wedge damper systems and the coupling between the longitudinal and lateral dynamics in the adapters - both through the action of the two-dimensional dry friction forces with stick-slip. Furthermore the effect of the tractive effort on the normal forces between the surfaces of the wedges is included. They give rise to an asymmetric distribution of the normal forces and the friction forces on one pair of the wedges on the end of the bolster.

The trucks carry a hopper wagon that runs on an ideal, straight and level track with constant speed. The model will be described with emphasis on its new features. The model for the dry friction dynamics in the damping system was formulated by True and Asmund [7]. It was specially developed for slow relative motion with stick-slip between plane, dry steel surfaces. Xia extended the one-dimensional model to the two-dimensional model used in this work.
We present the dynaraical system that models the dynamics of the moving wagon and show the result of numerical dynamical investigations such as the calculation of the critical speed, and the dynamics of the wagon on an irregular track and compare them with test results and simulation results using NUCARS.

Fujie Xia's $\mathrm{PhD}$-thesis [8] can be downloaded in its entity from the following web page: http://www.imm.dtu.dk/pubdb/views/publication_details. php?id $=1000$.

\section{THE WAGON MODEL}

We assume that the reader is so familiar with the design of the three-piece-freight truck that a resume of the new features in our dynamical model of the truck will be sufficient for the understanding of our analysis.

1 We consider two-dimensional dry friction forces in the contact surfaces in the adapters and the suubbers. It establishes a nonlinear coupling between the orthogonal components of the dry friction force in the surface.

2 In order to calculate the dry friction force in two dimensions in the plane of contact we introduce the friction direction angle and implement it into an algorithm. It repiaces the sign function used in the one-dimensional dry friction force computations.

3 The stick-slip motion between two bodies in contact - caused by dry friction - makes the state space of the system collapse. We therefore introduce switch conditions to treat the structure varying system. In the numerical routines the degrees of freedom of the system will change automatically depending on the magnitude of the friction force in the stick mode. 


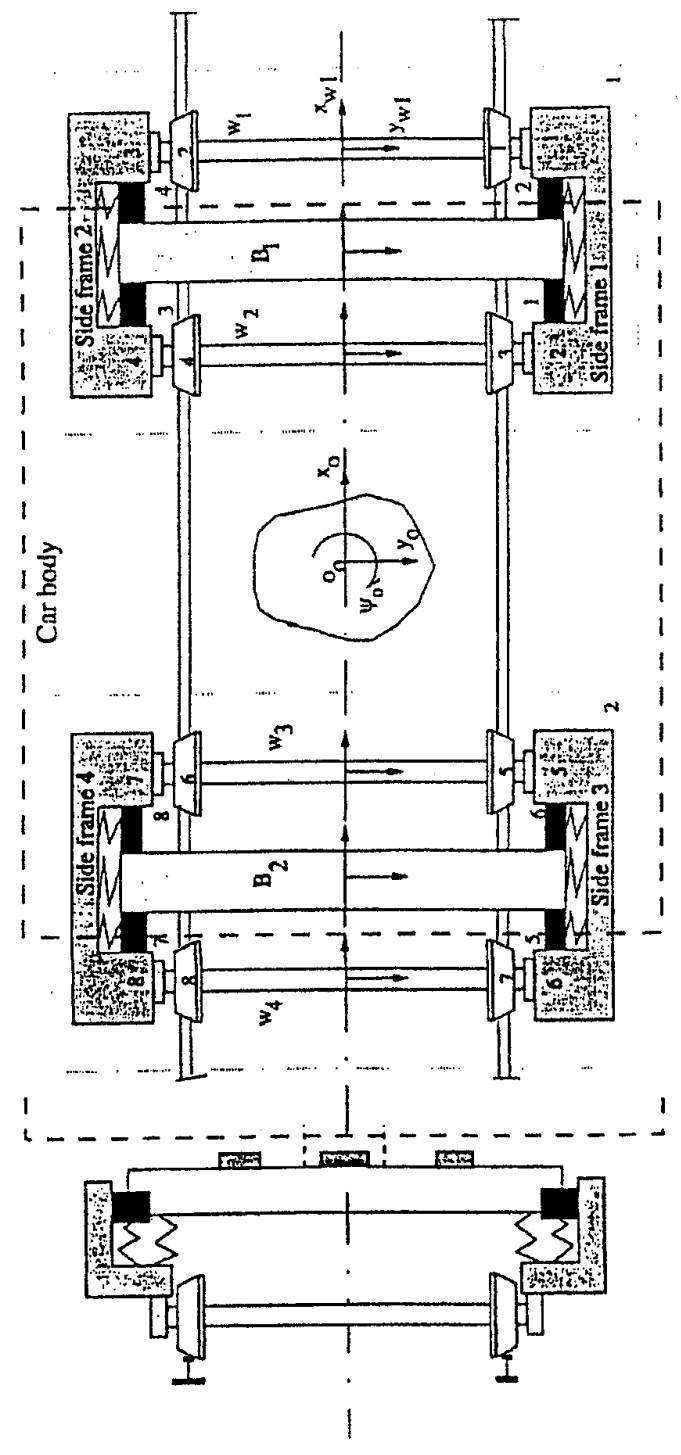

Figure 1: A diagram of the model of the wagon
Table 6.1: Degrees of freedom of the three-piece-freight-truck

\begin{tabular}{|c|c|c|c|c|c|c|}
\hline Components & Long. & Lat. & Vert. & Roll & Pitch & Yav \\
\hline Front truck leading wheelset & $u_{1}$ & $v_{1}$ & $w_{1}$ & $\widehat{\phi_{1}}$ & $x_{1}$ & $\psi_{1}$ \\
\hline Front truck trailing wheelset & $u_{2}$ & $v_{2}$ & $w_{2}$ & $\phi_{2}$ & $\chi_{2}$ & $\psi_{2}$ \\
\hline Rear truck leading wheelset & $u_{3}$ & $v_{3}$ & $w_{3}$ & $\phi_{3}$ & $\chi_{3}$ & $w_{3}$ \\
\hline Rear truck trailing wheelset & $u_{4}$ & $v_{4}$ & $w_{4}$ & $\phi_{4}$ & $x_{t}$ & $\psi_{4}$ \\
\hline Front truck left side frame & $u_{f 1}$ & $v_{s !}$ & $w_{f 1}$ & - & $x / 1$ & $\psi_{11}$ \\
\hline Front truck right side frame & $u / 2$ & $v_{/ 2}$ & $w_{\rho 2}$ & - & $x / 2$ & $\psi / 2$ \\
\hline Rear truck left side frame & $u_{/ 3}$ & $v_{/ 3}$ & $w / 3$ & - & $x_{53}$ & $\psi, 3$ \\
\hline Rear truck right side frame & $u / 4$ & $v_{14}$ & $w_{/ 4}$ & - & $x_{f 4}$ & $\dot{\psi}_{f t}$ \\
\hline \multirow[t]{2}{*}{ Front truck left wedges } & $u_{d 1}$ & $v_{d 1}$ & $w_{d 1}$ & - & - & $\rightarrow$ \\
\hline & $u_{d 2}$ & $v_{d 2}$ & $w_{d 2}$ & - & - & - \\
\hline \multirow[t]{2}{*}{ Front truck right wedges } & $u_{d 3}$ & $v_{d 3}$ & $w_{d 3}$ & 一 & - & 一 \\
\hline & $u_{d A}$ & $v_{d A}$ & $w_{d 4}$ & - & - & - \\
\hline \multirow[t]{2}{*}{ Rear truck left wedges } & $u_{d 5}$ & $v_{d 5}$ & $w_{d s}$ & - & - & - \\
\hline & $u_{d 6}$ & $v_{d \theta}$ & $w_{66}$ & - & 一 & $\longrightarrow$ \\
\hline \multirow[t]{2}{*}{ Rear truck right wedges } & $u_{d \tau}$ & $v_{d 7}$ & $w_{d 7}$ & - & - & - \\
\hline & $u_{d \theta}$ & $U_{d B}$ & $w_{\text {d8 }}$ & - & $一$ & 一 \\
\hline Front truck bolster & - & $v_{b l}$ & $w_{b 1}$ & $\phi_{01}$ & 一 & $\psi_{b 1}$ \\
\hline Rear truck bolster & - & $v_{b 2}$ & $w_{b 2}$ & $\phi_{\Delta 1}$ & - & $w_{b 1}$ \\
\hline Car body & - & $\nu_{0}$ & $w_{0}$ & $\phi_{0}$ & $x_{0}$ & $\psi_{0}$ \\
\hline
\end{tabular}

Figure 2: A table of the degrees of freedom

4 The effect of the tractive effort on the normal forces on the surfaces of the wedges is included.

5 Dead-band stops are introduced to describe the clearances between the side frames and the adapters in both the longitudinal and lateral directions as well as the clearance between the car body and the side supports.

6 The anti-warp stiffness is incorporated in the description of the three-piece-freight truck.

A diagram of our wagon model is shown on figure 1.

In order to model the stick-slip phenomenon we choose a velocity dependent friction force (see True and Asmund [7]). The friction force drops fast with growing relative speed from a 'stick value' to a 'slip value' given by Coulomb's friction law. In this way we avoid the numerical problem caused by a discontinuity, but we must introduce an unknown constant $\alpha$ that characterizes the drop rate. On the basis of several tests we have concluded that the dynamics is not sensitive to changes of the value of the constant within a reasonably wide range, and the dynamical results are thus 'robust'. On the basis of the tests the following model was selected. It is well suited for numerical implementation.

$$
F_{r}=F_{r, l} \operatorname{sech}(\alpha d x / d t)+F_{r, k}(1-\operatorname{sech}(\alpha d x / d t))
$$

$F_{r, l}$ is found from eqn.(10) if $|F| \leq B$ (see later) and. $F_{r, l}=(|F|-B)|F| / F$ if $|F|>B$,

$F_{r, h}=F-A|d x / d t| /(d x / d t)$,

$F_{r, l}$ is the acting friction force. It is unfortunately denoted by $F$ in equation $(10)$.

$\alpha$ is a scaling factor, which we have found it convenient to set to $50 \mathrm{~s} / \mathrm{m}$,

$F$ is the external shear force between the bodies in contact, and

$A=\mu N$ is the Coulomb friction force, $N$ the normal force

$B$ is the 'tear-loose-force' - the maximum value of the static friction force. 
With $B=1.2 \mathrm{~A}$ the friction force practically changes from $B$ to $A$ or back in the speed interval $[0,0.14 s]$.

Now we assume that the dry friction is isotropic and the contact surface between the two bodies is plane and smooth. Second we assume that the pressure between the two bodies is positive. In order to calculate a friction force vector on a plane we must find its modulus and argument in an appropriate $(x, y)$ coordinate system. When one of the bodies in contact moves with the relative velocity $V=\left(V_{x}, V_{y}\right) \neq(0,0)$ with respect to the other body, then the friction direction angle, is given by the angle between $V_{x}$ and $V$. The moduius of the friction force is then equal to the modulus of the normal force $|N|$ multiplied by the kinetic coefficient of friction, viz. $\left|F_{r}\right|=\mu_{k}|N|$. The direction of the friction force on each body is the direction of the relative velocity vector $V$ of the other body with respect to a local coordinate system in the contact plane of the body of interest.

If $V=(0,0)$ then we first replace all geometric constraints of the two bodies by reaction forces and torques. We approximate in this paper the effect of torques by the appropriate pair of forces in the contact planes. The friction force on each body is then equal to but in the opposite direction of the resiltant of the external forces on that body projected onto the contact plane. Although the bodies do not move relatively to each other, the friction force between them is not zero. This state is called a nonzero static friction force equilibrium state. It means that in this case the friction direction angle is determined by the argument of the projection of the external forces onto the contact plane. The maximum modulus of the friction force is equal to the modulus of the normal force $|N|$ multiplied by the static coefficient of friction, viz. $\left|F_{r, \max }\right|=\mu_{\mathrm{s}}|N|$.

When the relative velocities as well as the projection of the external forces onto the contact plane are equal to zero, then the friction force is also zero. This state is called a zero friction force equilibrium state. In this case the friction direction angle is undetermined.

The stick-slip motion will make the degrees of freedom of the system vary with time. This phenomenon is called collapse of the state space, and from several other investigations of simple mechanical systems we know that the phenomenon is very likely to make the dynamics of the system chaotic. There is no other method to handle this situation in a relatively simple way than to find all the switch conditions (see Eich-Soellner and Führer [1]) and the corresponding friction forces and treat the system as a piece-wise continuous one. The structure varying systems in the three-piece-freight truck are the subsytems of the bolster-wedge-frames and the bolster-frame-wheelsets. F. Xia in his thesis [8] chapter 4 shows how such systems can be handled by way of examples from the three-piece-freight truck design. He also makes numerical studies and presents a flow chart of the numerical algorithm for a variable degrees of freedom system. Here we present one simple one-dimensional dry friction problem as an illustration of the method.

Consider a body with mass $m_{2}$ on a plate with mass $m_{1}$. The plate is subjected to an excitation force $F_{1}$ and we have dry friction between the plate and the body. The situation is shown on figure 3.

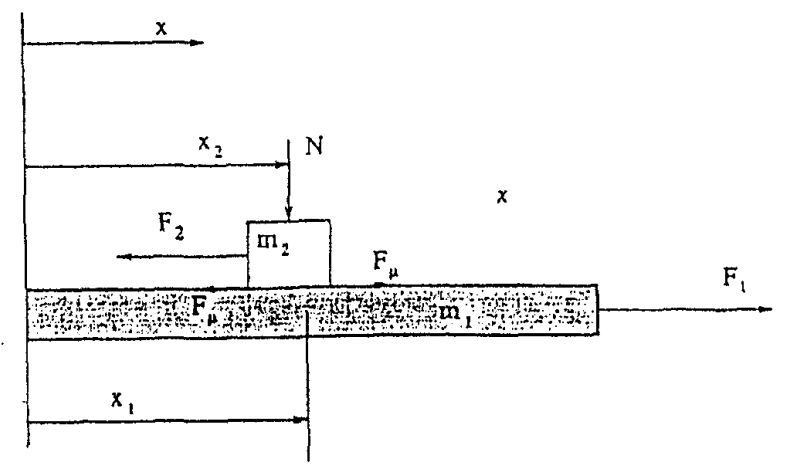

Figure 3: A body on a plate, which is excited by the force $F_{L}$

The equations of motion of the system can be written as

$$
\begin{aligned}
& m_{1} \ddot{x}_{1}=F_{1}-F_{\mu}, \\
& m_{2} \ddot{x}_{2}=F_{\mu}-F_{2} .
\end{aligned}
$$

The acting friction force is

$$
F_{\mu}=\mu N \operatorname{sign}\left(\dot{x_{1}}-\dot{x_{2}}\right)
$$

with $\mu$ as the kinematic coefficient of friction. If we solve the equations above we find that in the stick state the friction force will equal zero. That is not true because the acting friction force in the case of stick is zero only if $F_{2}=F_{1}=0$. As a consequence the numerical error will increase with increasing $\mu$ and the number of iteration steps will increase (see Eich-Soellner and Führer [1]). Let us therefore instead use the following method.

In the stick state the condition

$$
x_{1}-x_{2}=C
$$

holds. The first and second derivative of (4) with respect to time gives

$$
\begin{aligned}
& \dot{x_{1}}-\dot{x_{2}}=0 \\
& \ddot{x_{1}}-\ddot{x_{2}}=0 .
\end{aligned}
$$

When we substitute the equations (1) and (2) into equation (6) we obtain the switch condition for the stick motion

$$
F_{1} / m_{1}+F_{2} / m_{2} \leq F / m_{1}+F / m_{2}
$$

together with equation (5). $F$ denotes the static friction, which is different from zero. In this stick state the equations of motion of the system becomes

$$
\left(m_{1}+m_{2}\right) \ddot{x}=F_{1}-F_{2}, \quad \ddot{x_{1}}=\ddot{x_{2}}=\ddot{x}
$$

which means that the degrees of freedom of the system collapses to one. It can easily be verified that if the acting friction force for the stick-slip motion is correctly determined and the degrees of freedom were two to begin with then the system will change its degrees of freedom from two to one as shown here. In equation (7) set 

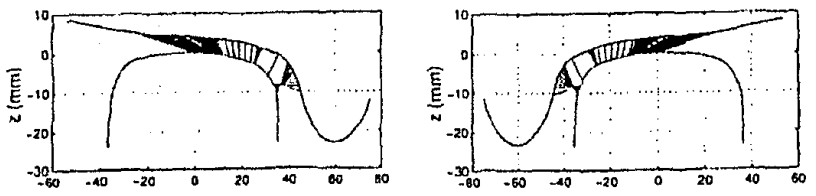

Figure 4: The possible contact points between the wheel profiles LM and the UIC60 profiles with a cant of $1 / 40$

$$
F_{1} / m_{1}+F_{2} / m_{2}=F / m_{1}+F / m_{2}
$$

then the acting friction force $F$ can be found to be

$$
F=\left(m_{2} F_{1}+m_{1} F_{2}\right) /\left(m_{1}+m_{2}\right) .
$$

If the acting friction force from $(10)$ is substituted into the equations (1) and (2) then the equations (1) and (2) become equal to equation (8). If any one of the conditions (5) and (7) is violated, then the acting friction force is determined by the kinematic friction coefficient multiplied by the normal force (eqn. (3)\}. Then the system returns to a two degrees of freedom system.

This example illustrates how a variable degrees of freedom system can be successfully modelled with our method.

We model a Chinese empty hopper wagon with threc-piecefreight trucks as a dynamical system. The derivation of the system is rather complicated and we must therefore refer the interested reader to Xia's $P h D$-thesis [8]. Its construction is very similar to North American wagons, but there exist small differences in the shape of the wedges and some parameter values. The wagon runs on UIC60 rails with a cant of $1 / 40$, and the wheel profiles are the Chinese LM profiles. The inside gauge between the flanges is $1353 \mathrm{~mm}$ in China. On figure 4 we show the possible rail/wheel contact points in dependence on the lateral displacement of the wheelset. The kinematic wheel-rail contact problem is solved numerically using Xia's routine WRKIN.

The contact is considered elastic so we can calculate the normal forces and the creep forces depending nonlinearly on the creepage. The normal forces are calculated by the staticdynamical method. First the static normal loads are determined with the instantaneous lateral displacement and yaw rotation of the wheelset. We use the static equilibrium equations with an iteration algorithm. Then the penetration caused by the dynamical forces along the normal to the tangent plane in the ideal contact point is calculated, and finally the normal forces are calculated using a Hertzian stiffness. For details please see Xia [8].

For the sake of comparison we have used the 'table looking method' based on Kalker's FASTSIM, Shen-Hedrick-Elkin's formula [5] and Polach's method [3] for the calculation of the creep forces.

The center plates, which connect the car body and the two bolsters, are modelled as spherical joints as shown on figure 5 . Because the radius of the spherical joint is small compared with the other dimensions of the car body we shall neglect the friction in the joint with exception of the effect on the yaw. In the equations of motion for the wedges we neglect the effect of torques. Between the side frames and the wheelsets we account for the state-dependent normal forces and dry friction on the contact

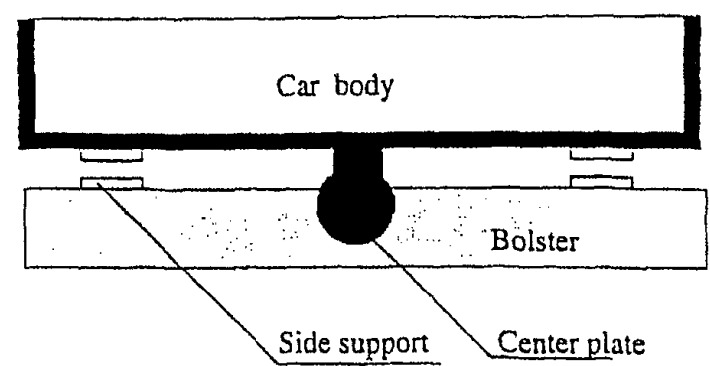

Figure 5: The center plate model

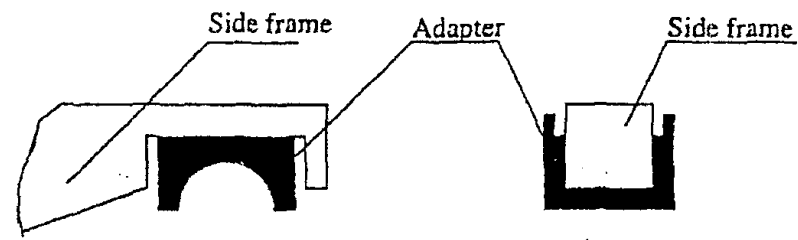

Figure 6: The contact between the end of a frame and an adapter

planes between the side frames and the wheelsets. Furthermore dead-band stops are used to model the clearances and the soft impacts.

All translations are blocked in the joint but the relative rotations are free. The clearances between the car body and the side supports are modelled as stiff springs with a clearance. The spring force act in the vertical direction, and the friction on the surfaces of the side supports are integrated in the yaw friction torque on the car body and the bolsters.

We allow for separation between the wedges and the bolster in the dynamical model.

The side frames are connected to the bolster through the wedges and to the wheelsets through adapters, where the adapter masses may be neglected. The yaw motion of the boister 'pushes' the side frames in the longitudinal direction. The vertical motions of the wheelsets will give rise to a corresponding vertical motion and a pitch rotation of the side frames. Therefore the connections between the side frames and the wheelsets (via adapters) are modelled as slide pairs as shown on figure 6. All the degrees of freedom introduced in our model of the hopper wagon are summarized in the table on figure 2.

\section{SOME RESULTS}

\subsection{THE WEDGE DAMPERS UNDER SINUSOIDAL EX CITATIONS}

We shall investigate two cases of the motion of the wedge dampers under controlled sinusoidal excitations of the bolster. First we consider the case when the system is started from a static equilibrium ie. it sticks. We then apply a lateral sinusoidally varying force of $5 \mathrm{kN}$ on the bolster with the frequency 


\begin{tabular}{|c|c|c|c|c|c|c|c|}
\hline & $2.5^{\circ}$ & $k_{d x}(N / m)$ & $0.8 \mathrm{e} 6$ & $k_{b y}(N / m)$ & 1.986 & & \\
\hline$\phi$ & $45^{\circ}$ & $k_{d y}(\mathrm{~N} / \mathrm{m})$ & $0.8 \mathrm{e} 6$ & $k_{b s}(N / m)$ & $2.69 \mathrm{cG}$ & $\mu_{\mathrm{k}, \mathrm{t}}$ & 0.35 \\
\hline$m_{d}\left(K_{B}\right)$ & 8 & $k_{d x}(\mathrm{~N} / \mathrm{m})$ & $1.0 \mathrm{e} 6$ & $I_{n d}$ & 0.4 & & \\
\hline$m_{b}(\mathrm{Kg})$ & $1906 / 10000$ & $k_{\delta z}(\mathrm{~N} / \mathrm{m})$ & $1.5 \mathrm{e} 7$ & $\mu_{k d}$ & 0.3 & & \\
\hline
\end{tabular}

Figure 7: The parameter values of the wedge damper system

of $8 \pi$. The hopper car is assumed to be empty. The parameters of the system are shown on figure 7 .

The lateral vibrations of the wedge and the bolster are bounded, periodic responses and the amplitude of the oscillations of the wedge is never larger than that of the bolster. Due to the dry friction damping the oscillation will reach a steady state with the same frequency as that of the excitation. The interesting feature is, however, that the lateral excitation also induces vertical motions of the bolster and the wedge. This phenomenon of parametric excitation can be explained in the following way: Before the lateral excitation the friction force on the surface between the bolster and the wedge has only a vertical component so the friction direction angle is $\pi / 2$. As soon as the lateral excitation starts, the friction direction angle changes from $\pi / 2$, which means that the lateral component of the friction force differs from zero so the resultant input force consisting of the excitation pius the gravity force acting on the bolster may be bigger than the total static friction force and then slip will occur. The vertical component of the friction force will drop to its kinematic value, the vertical balance is interrupted and the bolster accelerates in the vertical as well as in the lateral direction. The resulting oscillations are shown on figure 8 .

In the second case the bolster is sinusoidally excited in the vertical as well as in the lateral direction with the frequencies $4 \pi$ and $2 \pi$ respectively. In one subcase the amplitude is $3.5 \mathrm{kN}$ and in the other the amplitude is $5 \mathrm{kN}$. The initial condition is again one of static equilibrium. The resulting trajectories of the wedge and the bolster are shown on figure 9 .

\subsection{THE CRITICAL SPEED}

The critical speed is calculated as described by True [6]. We just recall that the critical speed is never determined alone by an eigenvalue analysis of the linearized dynamical problem. In the general case the critical speed is lower than the lowest value of the speed where the real part of a pair of eigenvalues of the linearized problem change sign from negative to positive. That value is called the Hopf bifurcation point, and it is the value of the speed where the investigated fundamental solution loses stability. The critical speed is the value of the speed where multiple attractors first appear. It is calculated by following all existing stable modes of motion - periodic as well as other forms - in state-parameter space - from the Hopf bifurcation point backwards ie. for decreasing speed to the value of the speed, where the only mathematical solution is the fundamental one we start out with. Please notice two important features of the threepiece-freight truck model: First a linearization around a basic solution does not exist due to the hysteresis loops and the discontinuities stemming from the dry friction and the impacts in the dead band stops. It means that no eigenvalue problem ex-
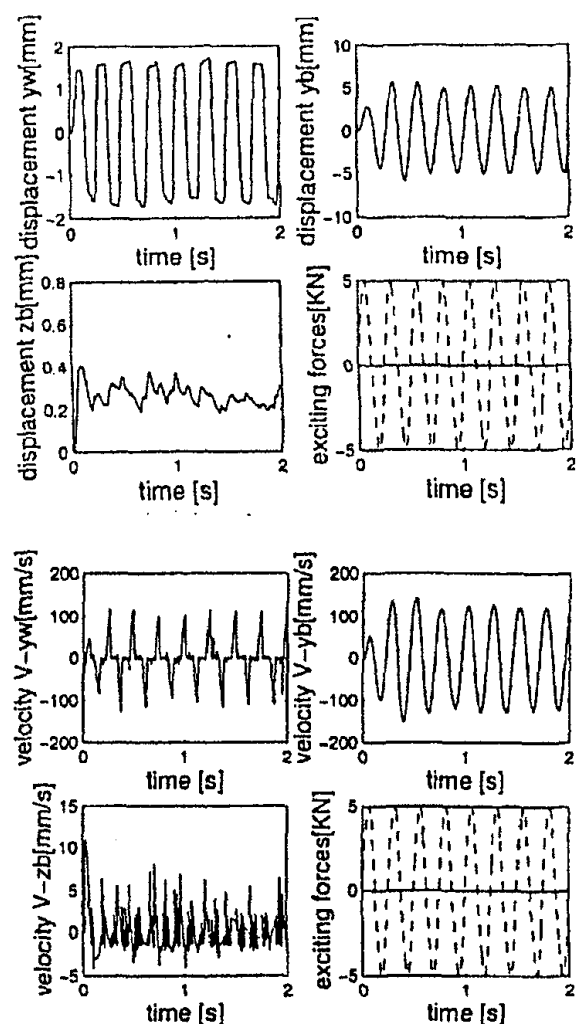

Figure 8: The lateral $(y)$ and vertical $(z)$ responses of a wedge and a bolster under purely lateral excitation on the bolster.
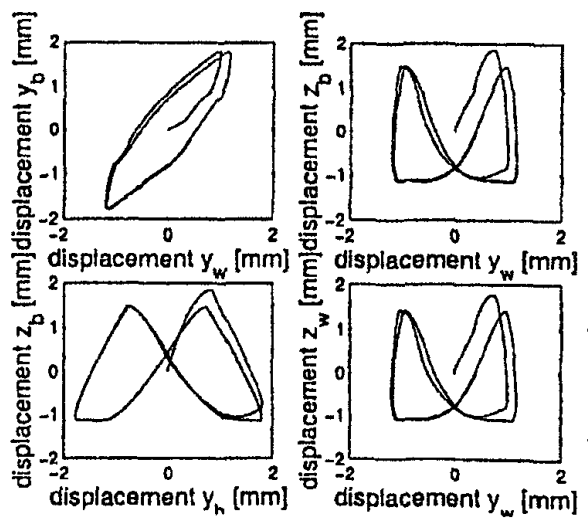

Figure 9: The trajectories of a wedge and a bolster under the combination of lateral and vertical excitations with the frequencies $2 \pi$ and $4 \pi$ respectively. The amplitude is $3.5 \mathrm{kN}$ on the left figures and $5 \mathrm{kN}$ on the right figures. 

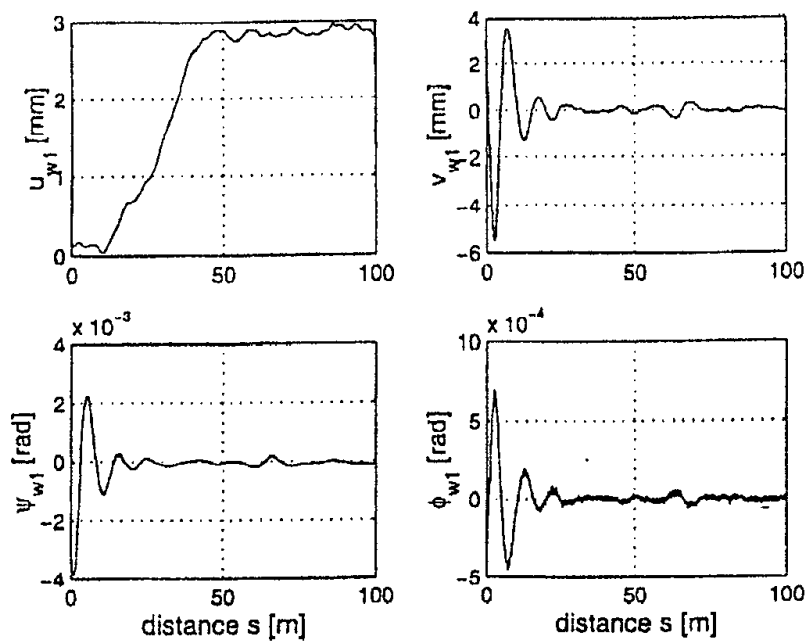

Figure 10: Longitudinal and lateral displacement, yaw and roll of the leading wheelset versus the distance at $\mathrm{V}=20 \mathrm{~m} / \mathrm{s}$.

ists! Second: The stick-slip action of the dry friction introduces - although it is deterministic - a sensitive dependence on the initial conditions - a hallmark of chaos - that makes 'a fundamental solution' non-unique. Time series plots of two solutions calculated with different initial conditions might look alike, but they will not yield the same set of state values at any time $t$.

We show the result of the calculation of the critical speed of the empty hopper wagon on bifurcation diagrams. A bifurcation diagram shows a characteristic measure of each and every solution of the dynamical problem versus a bifurcation parameter, which here is the speed of the hopper. In our case we only show the stable solutions - the socalled attractors. The track on which the hopper runs is straight, horizontal and ideal ie. free from disturbances. We start with a solution to the dynamical problem at a sufficiently low speed eg. $15 \mathrm{~m} / \mathrm{s}$. On figure 10 the state variables: Longitudinal and lateral displacement and yaw and roll of the leading wheelset are plotted versus the distance. It is obvious that the stable fundamental solution is not stationary it is not a fixpoint. The oscillations with a small amplitude look erratic and may be chaotic. The equilibrium in the $u$-coordinate oscillates near $3 \mathrm{~mm}$ and since the yow oscillates around 0 , the wheelset and the adapter moves ahead utilizing the play between the adapter and the side frame. This displacement is an effect of the stick-slip coming from the dry friction contact between the adapter and the side frame.

Next we increase the speed in sufficiently small steps and for every step we solve the dynamical problem using the end results of the preceding calculation as initial conditions for the next calculation. Around $\mathrm{V}=28.1 \mathrm{~m} / \mathrm{s}$ the fundamental solution loses its stability. The value can not be determined exactly due to the uncertainty stemming from the apparently chaotic nature of the fundamental solution. When we continue the process the transient will tend to move from the now unstable solution to the nearest attractor in the state space. It turns out to be a periodic
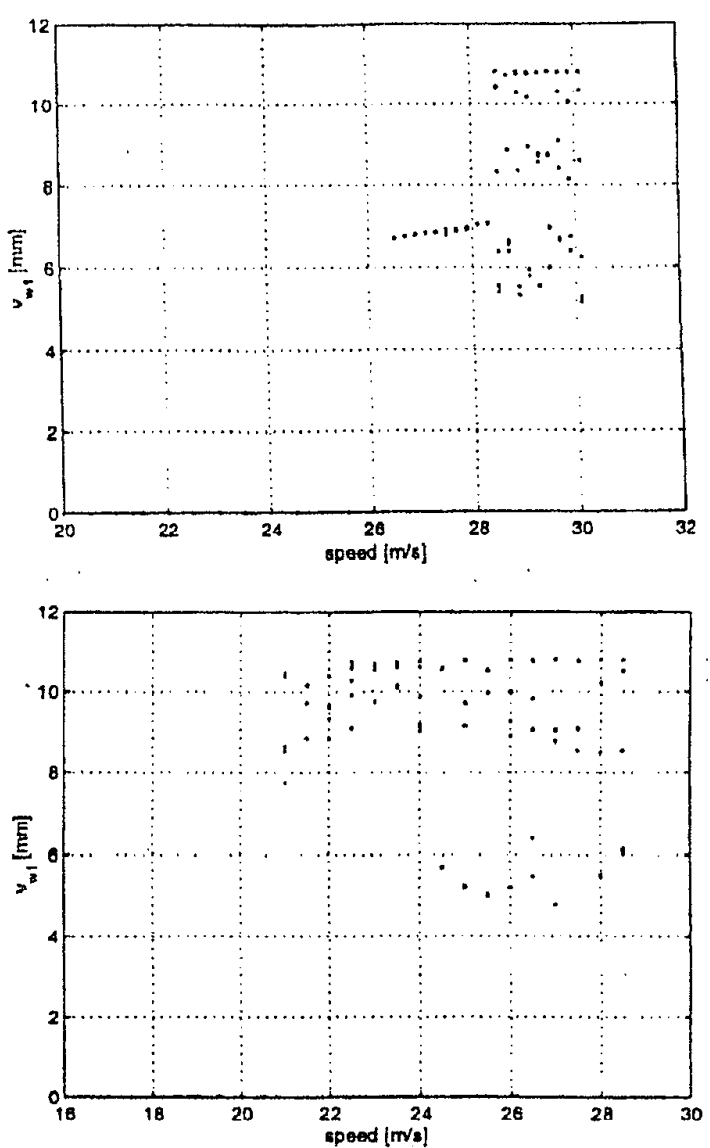

Figure 11: Bifurcation diagram of the three-piece-freight truck top for increasing speed and bottom for decreasing speed.

attractor. Shortly after at $\mathrm{V}=28.5 \mathrm{~m} / \mathrm{s}$ the periodic attractor loses its stability and only a chaotic attractor with rather high amplitudes of motion exists at least up to $V=30.0 \mathrm{~m} / \mathrm{s}$ where our investigation stops. Next we follow the chaotic attractor backwards in speed, and it dissappears rather abruptly at $\mathrm{V}=$ $20.5 \mathrm{~m} / \mathrm{s}$. Finally we go back to the periodic solution around $\mathrm{V}=28.1 \mathrm{~m} / \mathrm{s}$ and follows that backwards with the speed until it disappears at $\mathrm{V}=26.2 \mathrm{~m} / \mathrm{s}$. The bifurcation diagrams are presented on figure 11.

The result of the bifurcation analysis, which of course is performed numerically, is that the critical speed is $20.5 \mathrm{~m} / \mathrm{s} \sim$ $(46.1 \mathrm{mls} / \mathrm{h})$. Below that value only the fundamental solution exists. Between $20.5 \mathrm{~m} / \mathrm{s}<\mathrm{V}<26.2 \mathrm{~m} / \mathrm{s}$ two attractors exist: The fundamental solution and a chaotic solution. Between 26.2 $\mathrm{m} / \mathrm{s}<\mathrm{V}<28.1 \mathrm{~m} / \mathrm{s}$ three attractors exist: The two already mentioned and a periodic attractor. The periodic attractior can be seen as a set of points that lie on an almost linear curve on figure 11. Between $28.1 \mathrm{~m} / \mathrm{s}<\mathrm{V}<28.5 \mathrm{~m} / \mathrm{s}$ only two attractors exist since the fundamental solution now is unstable and above $\mathrm{V}=28.5 \mathrm{~m} / \mathrm{s}$ there is only one attractor, and it is chaotic. The scattered points on figure 11 indicate the chaotic attractor and 

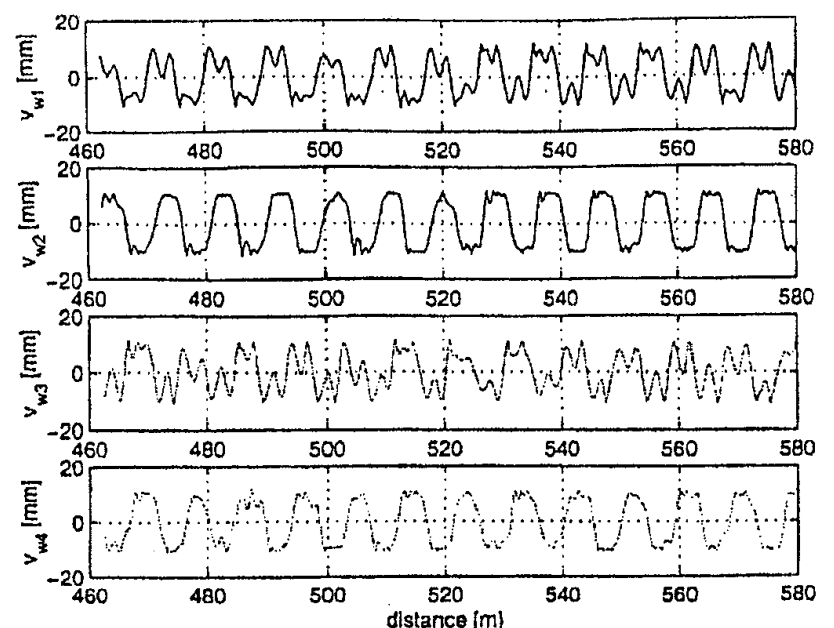

Figure 12: The lateral displacements of the wheelsets at $\mathrm{V}=29$ $\mathrm{m} / \mathrm{s}$.

the scatter is a measure of the amplitude variations. The specific mode of motion one might find in a road test in the speed range with multiple attractors depends on initial conditions and/or the size of the irregularities of the real track.

In order to verify the chaotic motion of the wagon we show on figure 12 the lateral displacements of the four wheelsets versus the running distance at $V=29 \mathrm{~m} / \mathrm{s} \sim 65.3 \mathrm{mls} / \mathrm{h}$ after the transients have died out. Although the motions are recurrent with a dominating frequency of approximately $3.1 \mathrm{~Hz}$ they are obviously not periodic. The chaotic nature is even more clearly seen on the plots on figure 13 of the projections of the phase space trajectories of the wheelsets $I$ and 2 . The axes on the four plots are lateral velocity $\dot{v}$ versus lateral displacement $v$ for wheelsets 1 and 2 respectively and angular velocity $\dot{\psi}$ versus yaw angle $\psi$ for wheelsets 1 and 2 respectively.

We have compared our results with a simulation using NUCARS. The result comes from China Academy of Railway Sciences [2] and it is shown on figure 14. The dynamical model is that of a gondola wagon with three-piece-freight trucks running at a speed of $22.778 \mathrm{~m} / \mathrm{s}$. It is a speed where we expect to find either the fundamental or chaotic motion but no periodic motion. Some parameters in the NUCARS calculation differ a little from the corresponding values of our hopper wagon. The most important difference between the two models, however, is the inclusion of the fully two-dimensional friction model, the stickslip motion and the structure varying systems in our model in contrast to the Chinese model that is analyzed with NUCARS. The Chinese result is a periodic motion after the transient - a kind of motion we do not find at that speed.

If the track has joints that excite the truck periodically, then the fundamental solution changes and a new bifurcation analysis with that motion as the fundamental one must be performed. From experience with other mechanical problems we would expect the chaotic attractor to dominate the behavior of the truck
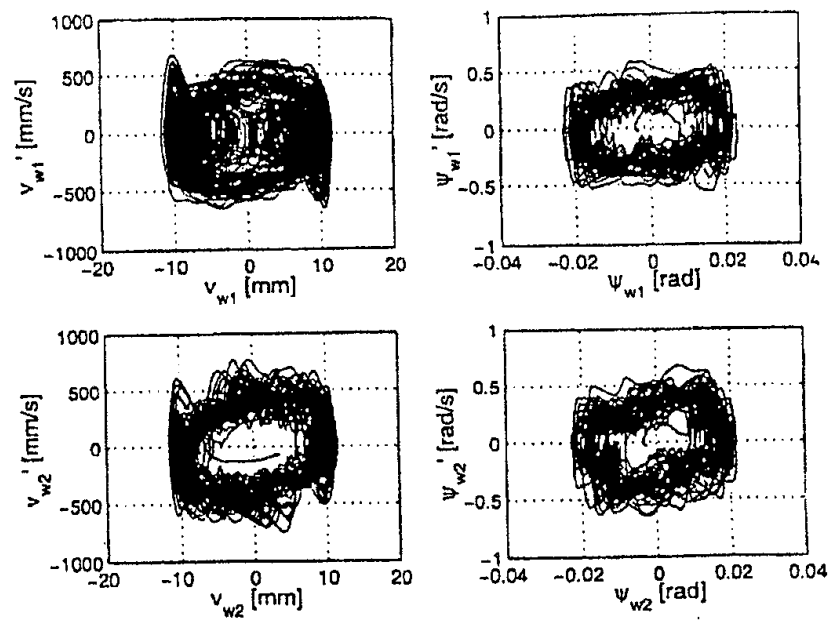

Figure 13: Phase plane projections of the state spaces of the wheelsets 1 and 2 at $V=29 \mathrm{~m} / \mathrm{s}$.

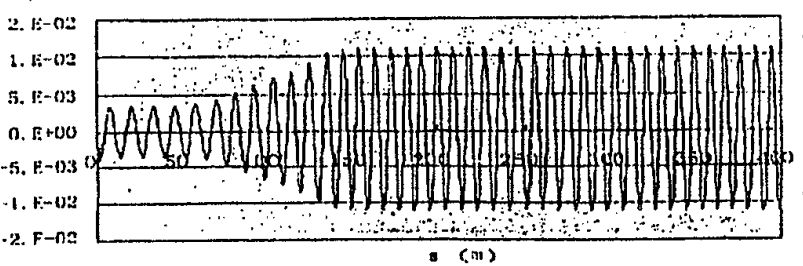

Figure 14: The lateral displacement of a wheelset calculated with NUCARS at $\mathrm{V}=22.778 \mathrm{~m} / \mathrm{s}$. 
in that case. The chaos may be created through mode interaction between two incommensurable frequences, one coming from the excitation by the track and the other from an eigenmode of the wagon, or from stochastic resonance if the excitation is not periodic.

\subsection{THE RESPONSE OF THE WAGON TO AN IRREG- ULAR TRACK}

It was our intention to simulate the dynamics of our hopper wagon running on a railroad in China and compare the results with measurements on a similar wagon running with the same speed on the same line. Unfortunately we were not able to obtain the track parameters we need as an input to our simulation. All we learned was that the railroad line where the measurements were taken is a 'class 6' railroad line with three power spectral densities (PSD) provided by the US Federal Railroad Administration. The PSDs characterize the vertical and lateral alignment and the track gauge and cross level. In our fully nonlinear analysis we cannot apply the PSDs directly as inputs, because the principle of superposition is not applicable to nonlinear problems. We must therefore first transform the PSDs into time series, which we may use as excitations of our wagon model. The transformation is described in Xia's thesis [8]. The calculated inputs do not of course correspond to the real inputs from the railroad line, because we have no information about the real distribution of the geometrical errors - a PSD only provides the frequency distribution of the data in terms of the spectral density of their mean square value. The comparison between the simulated and measured data can therefore give at best a qualitative agreement and never a point to point correspondance.

The transformation of a PSD to a time series introduces errors, and in order to estimate the errors we have transformed our time series back again into a PSD and compared the original PSD with the PSD obtained from the time series. As an example we show on figure 15 the original PSD on the same plot as 'the twice transformed' PSD for the vertical alignment, and the agreement must be characterized as being satisfactory.

We use the calculated time series of the track geometry in a simulation of the dynamics of the hopper wagon running on a class 6 railroad line with $78 \mathrm{~km} / \mathrm{h} \sim 48.75 \mathrm{mls} / \mathrm{h}$ - a speed higher than the critical speed of the hopper.

On figure 16 we show the measured lateral and vertical forces between the right front wheel and the rail and on figure 17 we show the result of our simulation. The measured forces oscillate more than the simulated ones. We believe the reason for this is that we use a new wheel profile in our simulation but the measurements were made on a hopper with a worn profile, and we were unfortunately not able to obtain the exact wheel profile of the wheels on that wagon.

On figure 18 we show the calculated motion of the leading wheelset at $78 \mathrm{~km} / \mathrm{h}$. Notice that the longitudinal motion of the wheelset is up to $2 \mathrm{~mm}$, so it should not be neglected in the calculations. When we compare the frequency of the dominating oscillations on figure 18 with the frequency of the oscillation on figure 14, which is found using NUCARS, we find a satisfactory agreement. The oscillations on figure 18 have a wavelength $\sim 9$

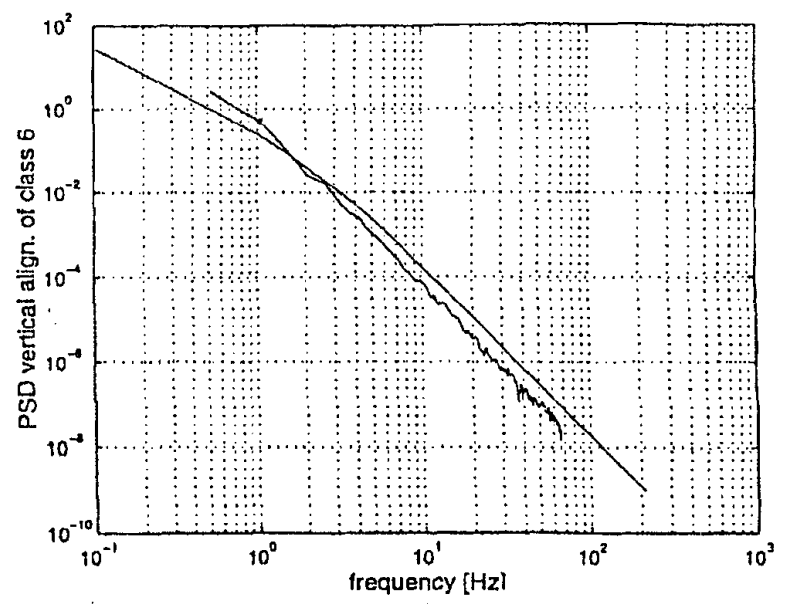

Figure 15: A comparison of the original (solid line) PSD of the vertical alignment of a railroad line of class 6 with our calculated one (marked with a star).

$m$ at the speed $22.67 \mathrm{~m} / \mathrm{s}$, which corresponds to a frequency of around $2.5 \mathrm{~Hz}$. The oscillation on figure 14 has a wavelength $10.5 \mathrm{~m}$ at the speed $22.78 \mathrm{~m} / \mathrm{s}$ corresponding to a frequency of around $2.2 \mathrm{~Hz}$.

\section{CONCLUSION}

With this new model of the dynamics of the three-piecefreight truck it should be possible to make more accurate simulations of the truck. The effects of the actual direction of the dry friction forces in the contact surfaces and of the stick-slip of the dry friction are nonlinear couplings between the motions of various elements, the forces and torques between them and their corresponding orthogonal components. The switch conditions are provided. Furthermore the asymmetric distribution of normal contact forces on the surfaces of the wredges due to the tractive effort is included. These effects have been neglected in earlier simulation programs. Stick-slip and the play in certain connections in the construction make the dynamical system a structure varying system. The structure varying property is known in mechanics to produce chaotic dynamics. We claim that the oscillations found in most of the investigations in Xia's thesis [8] support this assertion. Our results show that the chaos most often is seen as small amplitude disturbances on a basic motion. The basic motion may be a fixpoint in a coordinate system moving with the wagon along the center line of the track, if the speed is sufficiently low, or it may be a time periodic oscillation at higher speeds. In a few cases we have found sharp amplitude peaks that are instantaneously close to or larger than the dynamic safety limits imposed on that quantity. The relevance hereof in relation to railroad safety is an issue that should be dealt with by the proper authorities.

As long as the amplitudes of the oscillations are sraall, the chaos in itself is no more dangerous for railroad safety than small 

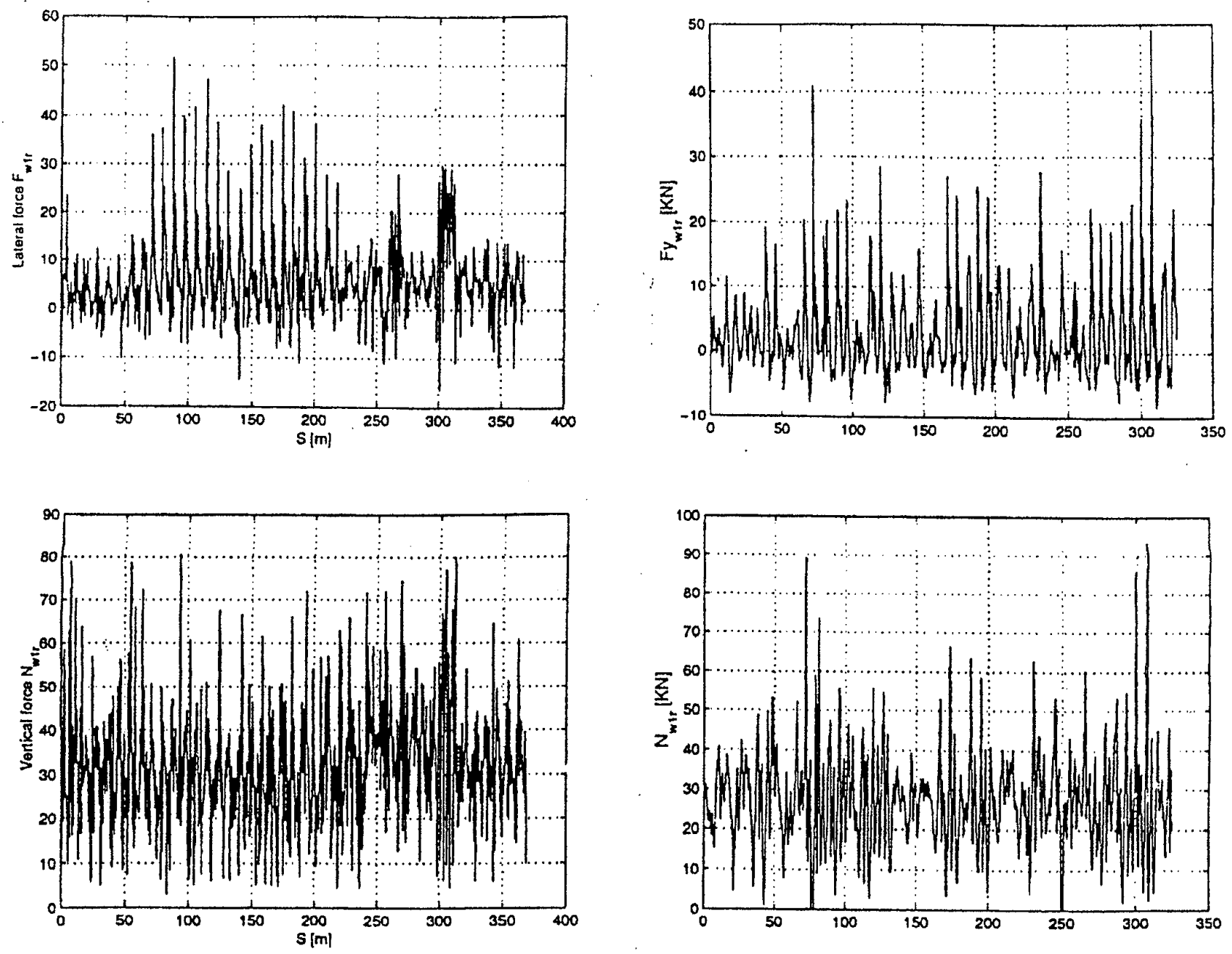

Figure 16: The measured lateral (top) and vertical (bottom) forces between the right front wheel and the rail.

Figure 17: The simulated lateral (top) and vertical (bottom) forces between the right front wheel and the rail. 

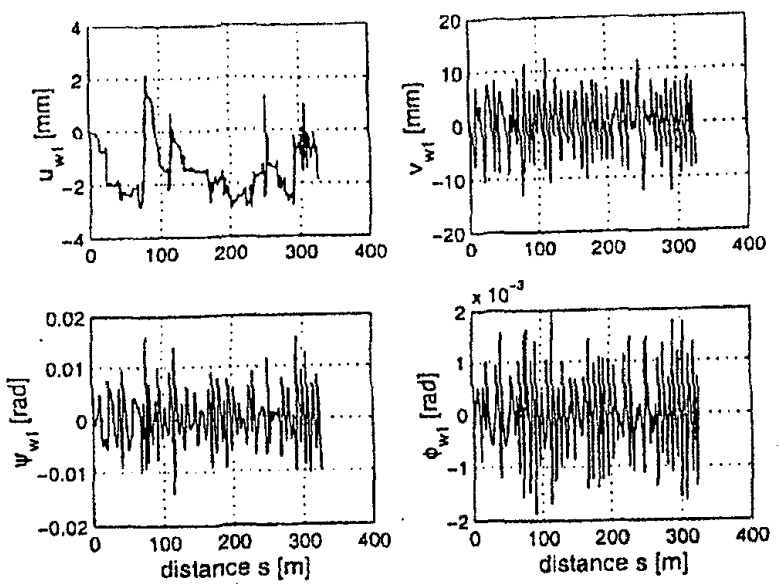

Figure 18: The motion of the front wheelset on an irregular track. $u, v$ denote the longitudinal and lateral displacernents; $\phi, \psi$ denote the roll and yaw rotations.

amplitude periodic oscillations are. Therefore there is no need to worry about the chaotic dynamics at speeds lower than the critical speed as described by True [6]. This conclusion does not hold for speed ranges where multiple attractors exist. When multiple attractors exist - for speeds above the critical speed then the transient motion from one attractor to another is often chaotic with very large peaks in the amplitudes. When the motion is chaotic to start with, then the transient will definitely be chaotic and may have dangerously high amplitudes. The study of chaotic transients is on the forefront of research in nonlinear dynamics today, and it is too early to draw drastic conclusions now on the basis of today's experience.

It is however important to consider the implications of the nature of chaos on simulations of the wagon dynamics. If you want to verify a simulation program by comparison with measured test results from a test with the vehicle on a railroad line then you should be able to identify the effects of isolated track geometry errors on the measured as well as the simulated results. The results most likely will not be exactly repeatable due to the chaotic dynamics of the correctly executed simulation as well as of the railroad vehicle and it is not to be expected that the values will be the same! The effects of non-isolated small scale disturbances may however vary greatly. This lies in the nature of chaotic dynamics. One of the definitions of chaos is that a chaotic motion is sensitive to variations of the initial conditions. The implication hereof is that no two series of experimental measurements of a chaotic motion will yield the same results because the initial conditions can not be controlled with an infinitely high accuracy, and the slightest difference leads to initially exponential divergence between two series of measurements. The same holds of course for the simulations. Chaotic motion is deterministic in the sense that two numerical simulations of the same situation using the same program, the same model and the same initial conditions with the same processor on the one and same computer will yield identical results in all time intervals, but if just one of the furst conditions is violated then the results will be significantly different after a certain - usually not long - time. If you use two different computers the results will differ if the processor and the hardware are not exactly the same and we have evidence (Jens Chr.Jensen personal communication) that the results may differ, when the calculations are performed on two different computers of the same brand but manufactured in two consecutive years and all other parameters are identical. Siuce it is impossible to determine any initial condition with arbitrarily high accuracy then it is simply impossible to achieve complete agreement between any test measurement series and a corresponding simulation when the dynamics is chaotic.

But is the real dynamics of the real three-piece-freight truck chaotic? Many - using the most accurate models - numerical investigations of the dynamics of the three-piece-freight truck indicate that its dynamics is chaotic, and the lack of exact repeatability of the measured test results support that conclusion. In an interesting paper Martin Schroeder [4] challenges that claim. He analysed truck hunting data very carefully for the existence of chaotic vibration. He applied various methods of analysis and his plots look very much like the same kind of plats made by us and support the existence of chaotic motion. Schroeder correctly states that the dimension of the phase space (in our moriel the dimension is $162=2.81$ ) 'is so large (that) even a 3-dimensional plot may not easily depict the true spatial structure, if indeed a structure exists'. Schroeder [4] therefore in addition calculates the maximum Lyapunov exponent, which is a measure of the divergence of the trajectories in the phase space starting from two adjacent initial conditions. From his results he correctly concludes that 'the existence of chaotic behavior does not seem prevalent for this vehicle operating under a hunting condition'. In another case Schroeder [4] writes that 'although (the maximum Lyapunov exponent) is positive, (less than .005), (it) is not considered significant for prediction of the existence of chaos'. This statement remains unchallenged by us since the CPU time needed for a calculation of the maximum Lyapunov exponent with our routine is very long, so we did not compute it.

Interestingly enough Schroeder's and our conclusions are not mutually exhaustive. As earlier mentioned our results gezerally display small amplitude chaos superposed on another type of motion. A great accuracy and high resolution in a big collection of data are therefore required in order to estimate the maximum Lyapunov exponent correctly. That can be obtained with great effort both in road tests and with simulations, but it has not yet been done. In this spirit Schroeder [4] critizises his own results in the last section and says that his conclusion is based on a data set that spans a relatively short time length. 'The differential vector lengths used in the Lyapunov exponent calculations needed to be larger than expected'. His calculations therefore may not have captured the chaos, which seems to contribute only little to the overall motion.

We therefore conclude that the chaotic part of the motion in the stationary case - contributes only very little to the track forces. The lack of predictability is the main effect of cliaos in that case. The transients, however, that are produced by singular track irregularities or occur spontaneously in the speed range 
with multiple attractors, may violate the safety standards, especially when the underlying dynamics is chaotic. The violations will be isolated events with no influence on the value of the maximum Lyapunov exponent. This conclusion is supported by Schroeder's last statement [4]. We agree with Schroeder that further investigations are highly recommendable.

The calculated value of the critical speed $(46.1 \mathrm{mals} / \mathrm{h})$ for the empty hopper wagon compares favourably with real life experience.

In the light of this discussion of chaos and under the conditions stated in section 3.3 the result of the calculation of the motion of the wagon on an irregular track can be termed 'satisfactory' and serve as a recommendation for the use of this model in other cases. Then the numerical solvers must be selected with the same care as we have executed, because they must be able to handle the chaotic dynamics correctly.

\section{REFERENCES}

[1] E. Eich-Soeliner and C. Führer. Numerical Methods in Multibody Dynamics. B. G. Teubner, Stuttgart, 1998.

[2] G. Li. Measuremental report on the derailment of wagons with three-piece-freight trucks on straight line. Technical Report TY 1408, China Academy of Railway Sciences, 2000.

[3] O. Polach. A Fast Wheel-Rail Forces Calculation Computer Code, pages 728-739. Proc. 16th IAVSD Symposium on Vehicle System Dynamics, The Dynamics of Vehicles on Roads and Tracks. Swets \& Zeitlinger, Lisse, 2000.

[4] M. P. Schroeder. Testing for the Existence of Chaotic Vibration in Truck Hunting Data, pages 1-6. RTD-Vol. 13, 1997 International Mechanical Engineering Congress and Exposition, Dallas, Texas, November 16-21, 1997. American Society of Mechanical Engineers, United Engineering Center, 345 East 47th Street, New York, New York 10017, USA, 1997.

[5] Z.Y. Shen, J.K. Hedrick, and J.A. Elkins. A Comparison of alternative Creep-Force models for Rail Vehicle Dynamical Analysis, pages 591-605. Proc. 8th IAVSD Symposium on Vehicle System Dynamics, The Dynamics of Vehicles on Roads and Tracks. Swets \& Zeitlinger, Lisse, 1984.

[6] H. True. Does a Critical Speed for Railroad Vehicles exist?, pages 125-131. RTD-Vol. 7, Proc. of the 1994 ASME/IEEE Joint Railroad Conference, Chicago Ill., March 22-24, 1994. American Society of Mechanical Engineers, United Engineering Center, 345 East 47th Street, New York, New York 10017, USA, 1994.

(7) H. True and R. Asmund. The dynamics of a railway freight wagon wheelset with dry friction damping. Vehicle System Dynamics, 38:149-163, 2002.

[8] F. Xia. The Dynamics of The Three-Piece-Freight Truck. PhD thesis, The Technical University of Denmark, 2002. 